\title{
BMJ Open Efficacy of coenzyme Q10 in patients with chronic kidney disease: protocol for a systematic review
}

\author{
Yongxing Xu, Juan Liu, Enhong Han, Yan Wang, Jianjun Gao
}

To cite: Xu Y, Liu J, Han E, et al. Efficacy of coenzyme Q10 in patients with chronic kidney disease: protocol for a systematic review. BMJ Open 2019;9:e029053. doi:10.1136/ bmjopen-2019-029053

- Prepublication history and additional material for this paper are available online. To view these files, please visit the journal online (http://dx.doi. org/10.1136/bmjopen-2019029053).

Received 10 January 2019 Revised 19 April 2019 Accepted 24 April 2019
Check for updates

(c) Author(s) (or their employer(s)) 2019. Re-use permitted under CC BY-NC. No commercial re-use. See rights and permissions. Published by BMJ.

The 306th Hospital of Chinese PLA, Beijing, China

Correspondence to Dr Jianjun Gao; ga0306kidney@126.com

\begin{abstract}
Introduction Coenzyme Q10 (CoQ10) is a fat-soluble vitamin-like quinone that exerts antioxidative functions and is also an important factor in mitochondrial metabolism. Plasma concentrations of CoQ10 are depressed in patients with chronic kidney disease (CKD). CoQ10 supplement can reduce adverse cardiovascular events, improve mitochondrial function and decrease oxidative stress in patients with non-dialysis CKD and dialysis CKD. We performed this study as a systematic review to comprehensively assess the effect of CoQ10 supplement on patients with CKD.

Methods and analysis The present systematic review protocol is reported in accordance with the Preferred Reporting Items for Systematic Reviews and Metaanalysis Protocols guidelines. The MEDLINE, EMBASE and Cochrane library databases will be searched without language restrictions in December 2018. Two reviewers will independently screen the references in two stages: screening of the title/abstract and then of the full-text, to identify references meeting the inclusion criteria. A descriptive overview and tabular and/or graphical summaries will be generated, and directed content analysis will be carried out on the extracted data. Ethics and dissemination This systematic review will evaluate the efficacy and safety of CoQ10 in patients with CKD. Ethical approval is not required for this study. The results of this systematic review will be presented in relevant conferences and published in a peer-review journal. PROSPERO registration number CRD42019120201
\end{abstract}

\section{INTRODUCTION}

The mortality rate of patients with chronic kidney disease (CKD) increases with a decrease in the glomerular filtration rate. Cardiovascular diseases, such as coronary artery disease, congestive heart failure and sudden cardiac death, represent the leading cause of morbidity and mortality in patients with CKD, particularly those who have reached end-stage renal disease. ${ }^{1}$ Diabetes, hypertension and obesity are traditional risk factors for cardiovascular disease, which are all more prevalent in patients with $\mathrm{CKD} .{ }^{2}$ In addition to the traditional risk factors, oxidative stress is also a key contributor to the pathogenesis of atherosclerosis and other cardiovascular

\section{Strengths and limitations of this study}

The systematic review is non-commercial and will comprehensively summarise the effects of a CoQ10 supplement on cardiovascular, oxidative stress, inflammation, glucose metabolism, lipid profiles in patients with chronic kidney disease (CKD).

- The present systematic review protocol is reported in accordance with the Preferred Reporting Items for Systematic Reviews and Meta-Analyses Protocols guidelines.

- Our review benefits from a sensitive search strategy including key terms, synonyms and medical subject headings.

- Both patients with non-dialysis CKD and those undergoing dialysis are to be included.

- In cases where it is not possible to pool data through meta-analysis, we will present outcome data in a narrative way, which will be a likely limitation.

diseases in patients undergoing maintenance haemodialysis. ${ }^{3}$ Oxidative stress originates from multiple sources, including decoupling of the electron transport chain in the mitochondria and inflammation-mediated production of superoxide via NADPH oxidase. Oxidative stress, inflammation and endothelial dysfunction are detectable even at the very early stage of CKD. ${ }^{4}$

Coenzyme Q10 (CoQ10) is a fat-soluble vitamin-like quinone, also known as ubiquinone, that exerts antioxidative functions. CoQ10 transports electrons from complexes 1 or 2 to complex 3 in mitochondria. ${ }^{5}$ CoQ10 treatment decreases superoxide production in endothelial cells and improves cardiac capacity in patients with heart failure. ${ }^{6}$ Long-term therapy with CoQ10 can reduce major adverse cardiovascular events, and is safe and well-tolerated by the general population. ${ }^{7}$ Plasma concentrations of CoQ10 are depressed in patients with non-dialysis CKD, and in those undergoing dialysis. ${ }^{8}{ }^{9}$ Depleting CoQ10 leads to inefficient electron transport and increased reactive oxygen species production. CoQ10 supplementation may improve mitochondrial function 
and decrease oxidative stress in patients undergoing haemodialysis. ${ }^{710}$ CoQ10 may have potential for preventing and treating cardiovascular disease, improving cardiac function and decreasing oxidative stress in patients with CKD. CoQ10 may have favourable effects on cardiac function, hypertension, glucose metabolism, lipid profiles, inflammation and oxidative stress in patients with non-dialysis CKD and those undergoing dialysis, but the results remain controversial. One recent meta-analysis showed that CoQ10 supplementation significantly improves the metabolic profile of patients with CKD. ${ }^{11}$ However, no published study has systematically and comprehensively summarised the effects of CoQ10 on cardiovascular outcomes, inflammation, oxidative stress, glucose metabolism and lipid profiles.

The aim of this systematic review will be to systematically appraise the evidence regarding the effects of CoQ10 supplementation in patients with CKD.

\section{METHODS}

\section{Protocol design and registration}

This protocol is reported in accordance with the Preferred Reporting Items for Systematic Reviews and Meta-analyses Protocol checklist (online supplementary file S1). ${ }^{12}$ To minimise reporting bias, this protocol was previously registered with the International Prospective Register of Systematic Reviews (PROSPERO), a platform for the international registration of prospective systematic reviews, and assigned the registration number CRD42019120201 (available at: http://www.crd.york.ac.uk/PROSPERO/ display_record.php?ID=CRD4201912021). We started this study in December 2018 and anticipate to complete the study in August 2019.

\section{Eligibility criteria}

The included trials will meet the following criteria: (1) randomised controlled, quasi-randomised, non-randomised trial or observational study (S); (2) participants with CKD (P); (3) the intervention of interest was CoQ10 treatment (I); (4) the comparator was adult patients with CKD who did not receive the intervention $(\mathrm{C})$.

\section{Prespecified outcomes}

The primary outcomes will be cardiovascular effects, including:

1. Cardiac function and structure: left ventricular ejection fraction (determined by echocardiography or contrast or radionuclide angiography); diastolic heart function; cardiac structure (measured by individual trials);

2. Biomarkers of cardiac function, such as brain natriuretic peptide and N-terminal-pro-b-type natriuretic peptide;

3. Blood pressure and heart rate;

4. Symptom improvement (measured by individual trials and/or by exercise capacity), quality of life (measured by individual trials);

5. Major cardiovascular event (cardiovascular mortality, non-fatal myocardial infarction, non-fatal stroke and re-vascularisation procedures).
Secondary outcomes of interest include effects on oxidative stress, inflammation, glucose metabolism, lipid profiles, all-cause mortality, safety and tolerability.

\section{Database and search strategy}

Searches will be performed, with no date restrictions, of the MEDLINE via Ovid, EMBASE via Ovid and Cochrane Library (Cochrane Central Register of Controlled Trials) electronic databases, using relevant text words and medical subject headings, as follows: kidney diseases, renal replacement therapy, renal insufficiency, dialysis, predialysis, diabetic nephropathies, ubiquinone, ubidecarenone, coenzyme Q10, co-enzyme Q10 and quinone. The clinicaltrials.gov website will be searched for relevant studies that have been registered and completed but remain to be published. The reference lists of articles and other reviews retrieved during the search or known to the authors will be searched for relevant articles. Elsewhere (online supplementary file S2), the full electronic search strategy for MEDLINE through Ovid is presented. There will be no language restrictions.

\section{Records and data management}

All citations identified by our search strategy will be exported to EndNote X9, a bibliographic management software and duplicates removed. The screening of remaining citations will be conducted by using Endnote X9, too. The data extraction will be performed on Microsoft Excel 2016.

\section{Study selection and data extraction}

Two independent reviewers will assess the eligibility of the trials with a standardised approach. Discrepancies will be resolved by discussion with a third individual. Two authors will independently extract data, including baseline patient characteristics, follow-up duration, intervention, outcome events and adverse events using a standardised data collection form. Any further information required from the original investigators will be requested by written correspondence, and any relevant information obtained in this manner will be included in the review.

\section{Assessing the risk of bias}

Two authors will independently assess the risk of bias of the randomised controlled trials according to the standard criteria. Seven different bias domains, including random sequence generation (selection bias), allocation concealment (selection bias), blinding of participants and personnel (performance bias), blinding of outcome assessment (detection bias), incomplete outcome data (attrition bias), selective reporting (reporting bias) and any other potential biases will be categorised as low risk of bias, high risk of bias or unclear risk of bias. ${ }^{13}$ Observational studies will be evaluated with the Newcastle-Ottawa Scale. ${ }^{14}$

\section{Statistical analysis}

A meta-analysis will be performed if sufficient data are available. The results of dichotomous outcomes will be expressed as risk ratios (RRs) or ORs with 95\% CIs for individual studies. For outcomes measured by continuous 
scales of measurement, the mean difference and $95 \% \mathrm{CI}$ will be used. For trials with end points with zero events in the treatment arm, RRs will be calculated using a 0.5 cell correction. ${ }^{15}$ There is no reason to assume that the effects being estimated in the different studies will not be identical; therefore, the random-effect model is the most appropriate choice for most meta-analyses. ${ }^{16}$ Accordingly, a Dersimonian-Laird random-effect model will be used. ${ }^{17}$ The heterogeneity of treatment effects between studies will be investigated statistically using the $\chi^{2}$ test and $\mathrm{I}^{2}$ statistic. $\mathrm{I}^{2}$ values of $25 \%, 50 \%$ and $75 \%$ correspond to low, medium and high levels of heterogeneity, respectively. ${ }^{18}$ If heterogeneity exists and there are a substantial number of studies, subgroup analyses and meta-regressions will be undertaken. A sensitivity analysis will be performed to exclude low-quality trials. Funnel plots, Egger's regression asymmetry test and Begg's test will be used to evaluate publication bias. ${ }^{1419} \mathrm{~A}$ two-sided $\mathrm{p}$ value $<0.05$ will be regarded as significant for all analyses. All analyses will be calculated using Stata software (V.12.0; StataCorp, College Station, Texas, USA). When there are insufficient clinically homogeneous trials to perform a meta-analysis, we will present a narrative synthesis.

\section{Assessment of quality of evidence}

An assessment of the quality of the evidence for the primary outcome will be made in accordance with the criteria suggested by the Grading of Recommendations Assessment, Development and Evaluation (GRADE) Workgroup. ${ }^{20}$ The GRADE system rates the quality of evidence across studies as very low, low, moderate or high.

\section{DISCUSSION}

This protocol presents an explicit plan of a systematic review to identify and summarise studies reporting the effects of CoQ10 in patients with CKD. To assess the quality of the evidence, the GRADE guidelines will be applied. In cases where it is not possible to pool data through meta-analysis, we will present outcome data in a narrative way, which will be a likely limitation. The existing evidence may be insufficient to make some robust conclusions; however, the results of this systematic review will provide important additional information relevant to the design of future trials.

\section{Patient and public involvement}

Patients were not involved in the development of the research question, outcome measure and study design.

Contributors $Y X$ is the first author, $J G$ is the corresponding author; $Y X$ and $J G$ designed the study; YX, JL and EH will acquire data; $Y X$ and JG will analyse and interpret data; $Y X, J L, Y W$ and JG drafted the initial and final manuscript; all authors approved the final version of the manuscript.

Funding The authors have not declared a specific grant for this research from any funding agency in the public, commercial or not-for-profit sectors.

Competing interests None declared.

Patient consent for publication Not required.
Ethics approval No human subject participants will be involved. On completion of the analysis, we will prepare a manuscript for publication in a peer-reviewed journal and present the results at conferences.

Provenance and peer review Not commissioned; externally peer reviewed.

Open access This is an open access article distributed in accordance with the Creative Commons Attribution Non Commercial (CC BY-NC 4.0) license, which permits others to distribute, remix, adapt, build upon this work non-commercially, and license their derivative works on different terms, provided the original work is properly cited, appropriate credit is given, any changes made indicated, and the use is non-commercial. See: http://creativecommons.org/licenses/by-nc/4.0/.

\section{REFERENCES}

1. House $A A$, Ronco $C$. The burden of cardiovascular risk in chronic kidney disease and dialysis patients (cardiorenal syndrome type 4). Contrib Nephrol 2011;171:50-6.

2. Ravarotto V, Simioni F, Pagnin E, et al. Oxidative stress - chronic kidney disease - cardiovascular disease: A vicious circle. Life Sci 2018;210:125-31.

3. Longenecker JC, Coresh J, Powe NR, et al. Traditional cardiovascular disease risk factors in dialysis patients compared with the general population: the CHOICE Study. J Am Soc Nephrol 2002;13:1918-27.

4. Matsuyama $\mathrm{Y}$, Terawaki $\mathrm{H}$, Terada $\mathrm{T}$, et al. Albumin thiol oxidation and serum protein carbonyl formation are progressively enhanced with advancing stages of chronic kidney disease. Clin Exp Nephrol 2009;13:308-15.

5. Jankowski J, Korzeniowska K, Cieślewicz A, et al. Coenzyme Q10 - A new player in the treatment of heart failure? Pharmacol Rep 2016;68:1015-19.

6. Beyer RE. The participation of coenzyme $Q$ in free radical production and antioxidation. Free Radic Biol Med 1990;8:545-65.

7. DiNicolantonio JJ, Bhutani J, McCarty MF, et al. Coenzyme Q10 for the treatment of heart failure: a review of the literature. Open Heart 2015;2:e000326.

8. Mehmetoglu I, Yerlikaya FH, Kurban S, et al. Oxidative stress markers in hemodialysis and peritoneal dialysis patients, including coenzyme Q10 and ischemia-modified albumin. Int J Artif Organs 2012;35:226-32.

9. Gazdíková K, Gvozdjáková A, Kucharská J, et al. Oxidative stress and plasma concentrations of coenzyme Q10, alpha-tocopherol, and beta-carotene in patients with a mild to moderate decrease of kidney function. Nephron 2001;88:285.

10. Rivara MB, Yeung CK, Robinson-Cohen C, et al. Effect of Coenzyme $\mathrm{Q}_{10}$ on Biomarkers of Oxidative Stress and Cardiac Function in Hemodialysis Patients: The $\mathrm{CoQ}_{10}$ Biomarker Trial. Am J Kidney Dis 2017;69:389-99.

11. Bakhshayeshkaram M, Lankarani KB, Mirhosseini N, et al. The effects of coenzyme q10 supplementation on metabolic profiles of patients with chronic kidney disease: a systematic review and meta-analysis of randomized controlled trials. Curr Pharm Des 2018;24:3710-23.

12. Shamseer L, Moher D, Clarke M, et al. Preferred reporting items for systematic review and meta-analysis protocols (PRISMA-P) 2015: elaboration and explanation. BMJ 2015;350:g7647.

13. Higgins JP, Altman DG, Gøtzsche PC, et al. The cochrane collaboration's tool for assessing risk of bias in randomised trials. BMJ 2011;343:d5928.

14. Egger M, Davey Smith G, Schneider M, et al. Bias in meta-analysis detected by a simple, graphical test. BMJ 1997;315:629-34.

15. Friedrich JO, Adhikari NK, Beyene J. Inclusion of zero total event trials in meta-analyses maintains analytic consistency and incorporates all available data. BMC Med Res Methodol 2007;7:5.

16. Borenstein M, Hedges LV, Higgins JP, et al. A basic introduction to fixed-effect and random-effects models for meta-analysis. Res Synth Methods 2010;1:97-111.

17. DerSimonian R, Laird N. Meta-analysis in clinical trials. Control Clin Trials 1986;7:177-88.

18. Higgins JP, Thompson SG, Deeks JJ, et al. Measuring inconsistency in meta-analyses. BMJ 2003;327:557-60.

19. Begg CB, Mazumdar M. Operating characteristics of a rank correlation test for publication bias. Biometrics 1994;50:1088-101.

20. Guyatt GH, Oxman AD, Vist GE, et al. GRADE: an emerging consensus on rating quality of evidence and strength of recommendations. BMJ 2008;336:924-26. 